\title{
A RCT addressing the impact of delivery mode after asymptomatic obstetric anal sphincter injury. (Mini-commentary on BJOG-20-0320.R1)
}

\author{
Riikka Tähtinen ${ }^{1}$ and Rufus Cartwright ${ }^{2}$ \\ 1 Tampere University Hospital \\ ${ }^{2}$ Imperial College London
}

August 12, 2020

Mini-commentary on BJOG-20-0320.R1: Cesarean section in the second delivery to prevent anal incontinence after asymptomatic obstetrical anal sphincter injury: the EPIC multicenter randomized trial

An obstetric anal sphincter injury poses an important clinical dilemma for subsequent vaginal deliveries, which may be complicated by recurrent obstetric anal sphincter injury and / or worsening or de novo anal incontinence.

Recurrent obstetric anal sphincter injury has a similar incidence to primary obstetric anal sphincter injury (6.3\% vs 5.7\%), and similar associated risk factors including instrumental delivery with either forceps [OR $3.12,95 \%$ confidence interval (CI) 2.42-4.01) or ventouse (OR 2.44, 95\%CI 1.83-3.25), birth weight [?]4 $\mathrm{kg}$ (OR 2.29, 95\% CI 2.06-2.54) and previous fourth-degree tear (OR 1.7, 95\%CI 1.24-2.36) (Jha S, Parker V: Int Urogynecol J. 2016 Jun;27(6):849-57).

The risk of long-term anal incontinence is also related to the degree of sphincter tear. Women with a fourthdegree sphincter injury in the first delivery are at higher risk for anal incontinence compared to women with a third-degree injury (58.8\% vs. 41.0\%). (Jangö H et al. 2018 Feb;218(2):232.e1-232.e10. Am J Obstet Gynecol). Although primary caesarean may be protective against anal incontinence, the previous observational evidence is consistent in finding that adjusted odds of long-term anal incontinence do not differ significantly by mode of second delivery after obstetric anal sphincter injury, and specifically that subsequent elective cesarean delivery is not protective (Jangö $\mathrm{H}$ et al, Am J Obstet Gynecol. 2016;214(6):733.e1-733.e13.) However, previous observational studies may suffer from confounding by indication, due to widespread adoption of planned caesarean for subsequent deliveries in women with incontinence symptoms or persistent sphincter defects

There have been no previous randomised trials to test whether anal incontinence could be prevented by planned cesarean section for the second delivery. Abramowitz and colleagues' (Abramowitz L et al. BJOG 2020) RCT provides us with a better understanding of the role of caesarean in women with asymptomatic third degree anal sphincter injury. There was limited cross-over between groups: of the 112 women randomized to the vaginal delivery group, $17(15.6 \%)$ had a caesarean section for obstetric indications. For those randomized to the planned cesarean section, 18 (16.58\%) delivered vaginally. One fifth of the randomized women did not complete the post-partum follow-up, but their characteristics did not differ between the two study groups. In this RCT, planned cesarean section in the second delivery was unequivocally not protective against anal incontinence at 8 months post-partum, with low rates of symptoms in both groups (Vaizey score $1 / 24$ vs. $1 / 24 \mathrm{p}=0.34$ ). As rates of incontinence were lower than expected, the trial may have been underpowered for a clinically relevant difference between groups. In an unplanned analysis, there was however, an 
interaction between baseline Vaizey score, and worsening symptoms after vaginal delivery, with significantly worse symptoms after vaginal delivery for women with pre-existing mild symptoms.

The authors rightly suggest that the findings are useful when counseling women about risks and benefits of caesarean at their second delivery. These results do not support advising systematic cesarean after asymptomatic third degree obstetric anal sphincter injury. The medicalization of pregnancy associated with planned caesarean is undesirable from both individual and societal perspectives, and cesarean delivery is associated with a number of health risks when compared to vaginal delivery (NICE Clinical Guideline CG132, https://www.nice.org.uk/guidance/cg132/). Important questions remain for future work whether subsequent cesarean section may be useful in the long term, among women with mildly symptomatic anal incontinence, or for women with asymptomatic fourth degree obstetric anal sphincter injury.

Disclosure of interests: Tähtinen declares honoraria from Olympus. Cartwright declares no conflicts of interest. Completed disclosure of interest forms are available to view online as supporting information. 